Produto \& Produção, vol. 13 n. 2, p. 95-105, jun. 2012

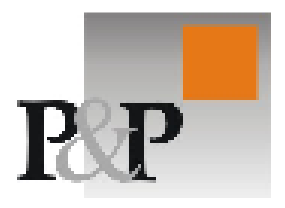

\title{
Administração de estoques: diferentes formas de medição da acuracidade
}

Recebido em 30/03/2011. Aceito em 09/02/2012.

\section{Fabio Favaretto}

Universidade Federal de Itajubá - UNIFEI

fabio.favaretto@unifei.edu.br

Este artigo trata de falta de padronização da informação da acuracidade de um estoque. O objetivo é apresentar diferentes formas de medição da acuracidade de estoques e fazer considerações a respeito destas medidas. Falhas na contagem e no registro das movimentações do produto incidem no sistema de controle deste estoque. Foram analisadas a diferença média dos saldos dos produtos em quantidade e em proporção e o número de produtos com saldos distintos no controle e no estoque real. Foram criados cenários com diferentes probabilidades de ocorrência de erro em cada movimentação do estoque. Para cada cenário foram consideradas situações distintas em relação à tolerância ao erro: sem tolerância e com tolerâncias de 1 e $5 \%$. Para que o decisor envolvido na gestão de um processo de controle de estoque tenha uma boa visão da acuracidade do mesmo, sugere-se a utilização conjunta das informações das medidas da diferença proporciona e a medida do número de produtos que apresentam diferença entre os saldos.

Palavras-chave: acuracidade de estoques, controle de estoque, planejamento da produção.

This article shows the lack of standardization in an inventory accuracy measure. The goal is to present different ways of measuring accuracy of inventories and make considerations concerning these measures. Counting and controlling problems in product inventory registry bring problems to inventory control. There were analyzed the medium difference in terms of products and rate besides the number of products with different inventories. Scenarios with different probabilities for error occurrences were created. Each scenario considered distinct error tolerance: 0,1 and $5 \%$ tolerance. As conclusion it is suggested that inventory managers use more than one measure at a time.

Keywords: inventory accuracy, inventory control, production planning. 


\section{INTRODUÇÃO}

O bom controle dos estoques de uma empresa é uma atividade essencial para sua competitividade. A falta de material em estoque pode fazer com que o nível de serviço seja comprometido e clientes deixem de ser atendidos. Por outro lado, excesso de material em estoque traz problemas de fluxo de caixa, espaço e perdas por obsolescência.

Um sistema de controle de estoque registra todas as movimentações de entrada e saída de materiais, assim como acompanha o saldo deste para que seja feita a decisão de pedir ou não uma reposição quando o nível está baixo. Caso exista algum tipo de erro neste controle, os pedidos podem ser feitos sem necessidade ou então não serem feitos pedidos quando estes são necessários. Algumas das causas dos erros no registro e movimentação de estoques são: i) erros de contagem ou de digitação, ii) deixar de registrar movimentações de entrada ou saída, iii) roubo de material, iv) mais de um controle para o mesmo material e v) perda ou extravio de material.

A exatidão com que um sistema de controle de estoque representa o estoque real é medida através da acuracidade dos registros de estoque. Basinger (2006) apresenta que a falta de acuracidade de dados do estoque acarreta na redução do nível de serviço e aumenta os custos relacionados às atividades da logística. $\mathrm{Na}$ mesma linha, Huschka (2009) destaca que a precisão dos saldos de estoque é um fator crítico para o bom desempenho das atividades industriais.

Muitas organizações determinam níveis e metas para a acuracidade de seus estoques. Para isso, devem gerenciar informações relativas a esta acuracidade. Esta informação é o objeto de pesquisa deste artigo.

A pesquisa acadêmica tem, como um de seus objetivos, auxiliar as organizações a atingirem melhores resultados que permitam vantagens competitivas ou as qualifiquem para a concorrência. Um dos focos de pesquisa que se destaca atualmente é o processo decisório. Como suporte a este processo também é estudado o gerenciamento das informações, tido como um dos principais bens de uma organização. Informações são um dos componentes do processo de decisão.

O estudo do papel da informação no processo decisório foi concentrado nos anos 80 e 90 do Século passado em termos de Sistemas de Informações (SI) e sua participação neste processo. Assim, surgiram termos como Sistemas de Informação Gerenciais e Sistemas de Apoio à Decisão, entre outros. Grande parte dos estudos relacionados a estes sistemas teve foco na tecnologia, e não nas necessidades de informação dos usuários, no caso os decisores de uma organização. Atualmente, se discute sobre a validade deste tipo de solução, visto que a tecnologia apenas disponibiliza as informações aos usuários.

Saber qual informação usar para se tomar uma decisão, e de que forma a apresentar tem sido o foco de alguns estudos recentes. Reid e Catterall (2005) afirmam que os problemas de qualidade de dados recebem pouca atenção na literatura, e que este é um fator que pode melhorar a efetividade das decisões tomadas. Para Guimarães e Évora (2004), a maneira como a informação é obtida, organizada, gravada, recuperada e posteriormente utilizada permite ao gerente atuar com mais segurança, aumentando a possibilidade de acerto na tomada de decisão.

Apesar da importância de se saber exatamente (acuradamente) a quantidade dos materiais disponíveis em estoque, nem sempre é possível se ter esta informação com a qualidade adequada. Em Klein (2001), se afirma que não existem 
bancos de dados corporativos com $100 \%$ de acuracidade em todos os dados. Assim, pode-se esperar que em bancos de dados de controle de estoques também existam erros que comprometam a acuracidade das quantidades de materiais. Além destes erros inerentes aos próprios dados, existe uma falta de padronização quanto à forma de medir a acuracidade de estoques. Algumas formas podem não mostrar claramente o fenômeno que se quer conhecer, ou ainda serem claramente tendenciosas. Este artigo foi desenvolvido a partir deste problema de falta de padronização da informação da acuracidade de um estoque. O principal objetivo é apresentar diferentes formas de medição da acuracidade de estoques e fazer considerações a respeito disto.

A contribuição deste trabalho é apresentar como a informação relacionada à acuracidade de um estoque pode ser obtida de diferentes formas e como isto interfere nos processos de decisão relacionados à administração de materiais. Em diversas situações não é feita referência à forma como a acuracidade foi medida. Como será mostrado, a mesma situação real de controle de estoques pode apresentar diferentes medidas de acuracidade. Com este conhecimento, os gestores podem complementar suas decisões com informações adicionais e complementares.

A estrutura deste artigo é iniciada com esta introdução, a definição do problema de pesquisa e o seu objetivo. A seguir são apresentadas a metodologia utilizada e uma revisão da literatura sobre acuracidade de estoques. Feito isto, são apresentados os resultados obtidos e estes são analisados, permitindo a finalização deste trabalho com algumas conclusões.

\section{METODOLOGIA}

Para a realização deste artigo foi adotada uma metodologia de pesquisa exploratória. Pelo fato da literatura existente não definir claramente o objeto de pesquisa (informação da acuracidade de estoques), não é possível se fazerem análises descritivas ou experimentais. O resultado esperado é uma referência sobre possíveis formas de se medir a acuracidade de estoques e suas características. Por se tratar de uma pesquisa exploratória será feito o emprego de técnicas estatísticas para análise da significância de diferenças entre valores. Estas análises serão feitas com base em uma estatística descritiva.

Para se atingirem os objetivos propostos, a metodologia de pesquisa foi dividida em etapas, descritas a seguir.

A primeira etapa da pesquisa é a das definições iniciais do problema, objetivos e metodologia a ser utilizada. A simulação computacional foi a forma encontrada para se obterem os resultados pretendidos. Especificamente foi utilizada a simulação de Monte Carlo, por se tratar de um problema estático onde são necessárias repetições com números gerados aleatoriamente.

A segunda etapa é a revisão da literatura sobre o tema, especificamente sobre a acuracidade de estoques. A maior parte dos trabalhos encontrados neste tema trata da importância de altas acuracidades (próximas a 100\%) e formas de obtê-la. Com o conhecimento obtido nesta etapa foi possível a definição do ambiente de simulação e a criação dos cenários utilizados.

Feitas as etapas anteriores, a terceira etapa trata da definição da elaboração dos cenários que serão utilizados para se tecerem as considerações. Estes cenários serão construídos em planilha eletrônica MS Excel, com o auxílio do simulador 
Crystal Ball para geração de valores aleatórios. A planilha retrata a situação apresentada na Figura 1 a seguir. É feita uma movimentação aleatória de um material, que pode ou não ter erro. Estes cenários irão simular uma situação onde existe um estoque real (físico) e um estoque controlado por um sistema, sendo que este último pode conter erros. A comparação entre os controles dos estoques (real $X$ sistema) será a base para as considerações. Foi utilizado o modelo de controle de estoque por ponto de pedido, e a configuração dos parâmetros utilizados buscou a reprodução de uma situação corrente.

A quarta etapa consiste na geração dos cenários definidos anteriormente e das comparações necessárias. Estes cenários reproduzem situações encontradas na literatura onde existem diferentes tolerâncias para erros nos controles de estoques. Uma determinada tolerância é utilizada para que erros abaixo deste valor não sejam considerados.

A última etapa é a análise dos resultados e descrição das formas de medição da acuracidade de estoques, assim como a elaboração das conclusões. As simulações geram valores aleatórios para os parâmetros e indicadores para os resultados analisados. Neste trabalho se procurou uma visão descritiva dos resultados, através de sua média e dispersão.

\section{ACURACIDADE DE ESTOQUES}

Menores estoques e maiores níveis de serviço ao cliente são desafios constantes das empresas. No entanto, para quebrar este trade-off, tanto a academia como as empresas têm esbarrado em algumas barreiras. Uma dessas barreiras é a baixa precisão das informações relativas aos saldos de estoque. Pois quanto mais precisas forem as informações dos estoques, mais seguras serão as decisões de quanto e quando pedir os materiais para os fornecedores, por conseqüência melhorar o gerenciamento dos estoques (WALLER et al, 2006).

A redução nos níveis de estoque sem um nível satisfatório de qualidade de informação entre a quantidade física e a quantidade do sistema (acuracidade de estoque) pode acarretar em uma série de agravantes para os processos internos e externos da organização, além dos respectivos custos relacionados. Dois efeitos que a falta de acuracidade nas informações de estoques geram podem ser caracterizados como o aumento no tamanho do lote e a incerteza do atendimento da demanda (UÇKUN et al, 2008).

Ballard (1996) destaca a importância da precisão das informações de estoque, pois estas são usadas em diversos processos de uma empresa, como o planejamento da produção, logística e controle financeiro. O autor apresenta diversos aspectos que devem ser controlados e monitorados para garantir uma informação acurada, inclusive com contagens cíclicas e outros procedimentos de garantia para um sistema de controle de estoque.

Em uma análise de modelos de gerenciamento de estoques, Buxey (2006) aponta que, independentemente do modelo utilizado, deve ser feito um esforço contínuo (contagens e conferências) para se manter a acuracidade das informações sobre as posições dos estoques. Caso isso não ocorra, a eficiência de qualquer modelo é prejudicada. Feldmann e Müller (2003) e Klein (2001) colocam que a utilização de incentivos para os operadores envolvidos diretamente nos processos 
de controle de estoques apresenta bons resultados para garantir informações verdadeiras sobre as posições dos estoques.

Brown et alli (2001) apresentam um pesquisa onde os efeitos da falta de acuracidade de estoques são analisados no planejamento da produção. Os autores afirmam que não deve ser controlada apenas a acuracidade relacionada à quantidade de registros errados, mas também em relação à faixas de erros aceitos e também da localização de produtos nos estoques.

Zhang et alli (2006) destacam a importância das informações de posição dos estoques serem acuradas e estarem disponíveis quando necessárias em uma cadeia de suprimentos. Os autores relacionam essa disponibilidade à presença da tecnologia de informação e definem fatores de segurança para situações onde as informações de estoques não seguem padrões estabelecidos.

Fleisch e Tellkamp (2003) apontam através de um estudo de simulação que, até $10 \%$ dos lucros de uma empresa podem ser perdidos em situações com falta de acuracidade nos registros de estoques. Os efeitos decorrentes deste problema refletem no atraso de entregas de produtos a clientes, assim como no aumento dos custos de uma forma geral.

Uçkun et al (2008) destacam que a falta de acuracidade de estoque, interfere diretamente no tamanho do lote e na certeza do atendimento da demanda (UÇKUN et al, 2008).

\section{DEFINIÇÃO DOS CENÁRIOS E DAS FORMAS DE MEDIÇÃO}

Para realização desta pesquisa foi definido um cenário com movimentações em um determinado estoque, controlado através do modelo de Ponto de Reposição ou Ponto de Pedido. Neste modelo é definida uma quantidade de material que aciona um novo pedido para repor o material utilizado de forma a garantir que não haverá falta de material. Os valores utilizados na definição do modelo foram os mesmos para todos os cenários e são apresentados a seguir: i) saldo inicial: 2000 unidades; ii) ponto de pedido: 1000 unidades; iii) lote de reposição: 2000 unidades; iv) tempo de reposição: 2 dias; e v) movimentação diária: valor aleatório com distribuição discreta uniforme de probabilidade entre 0 e 200 unidades.

Considera-se apenas uma movimentação de saída diária, gerada aleatoriamente. Caso seja necessário e tenha sido feito o pedido de reposição anteriormente, pode existir uma movimentação diária de entrada. São considerados períodos de 30 dias, porém é considerado apenas o saldo do último dia, para que os valores iniciais não contaminem os resultados. As mesmas definições são utilizadas para caracterização do estoque de 1000 produtos distintos.

As definições acima são empregadas no controle do estoque de um material real, ou seja, sem erros. Possíveis erros de movimentação no controle de estoques são apresentados em Drohomeretski (2009) e em Favaretto (2010). A partir do controle de estoque real é gerada a possibilidade de erro em cada movimentação, como apresentado na Figura 1. Isso é feito através de uma variável aleatória binária (só pode assumir os valores 0 ou 1) que indica se o registro vai ter erro ou não. Foram feitos dois cenários: um com $1 \%$ e outro com $10 \%$ de probabilidade de erro em cada registro de movimentação de estoque. Caso a movimentação tenha erro, o valor deste erro também é gerado aleatoriamente através de uma variável com 
distribuição discreta uniforme com valores entre -100 e 100 unidades. Isso gera tanto erros positivos como negativos em cada registro.

Além destas configurações, foram consideradas situações com diferentes tolerâncias para os erros encontrados. Foram consideradas três situações: sem tolerância, com tolerância de $1 \%$ e de $5 \%$. Uma tolerância significa que erros inferiores ao valor especificado não são considerados. Para cada cenário analisado foram gerados 1000 produtos com as definições descritas acima.

Foram consideradas três formas de medição da acuracidade. Isso é feito medindo-se a falta de acuracidade, ou a inacuracidade. Uma inacuracidade de $0 \%$ indica que o controle do estoque é fiel ao estoque real. Ressalta-se que os principais dicionários da língua portuguesa não trazem a palavra "acuracidade", que seria uma quantificação da acurácia do controle do estoque. Entretanto esta palavra será utilizada por ser largamente empregada na comunicação corrente.

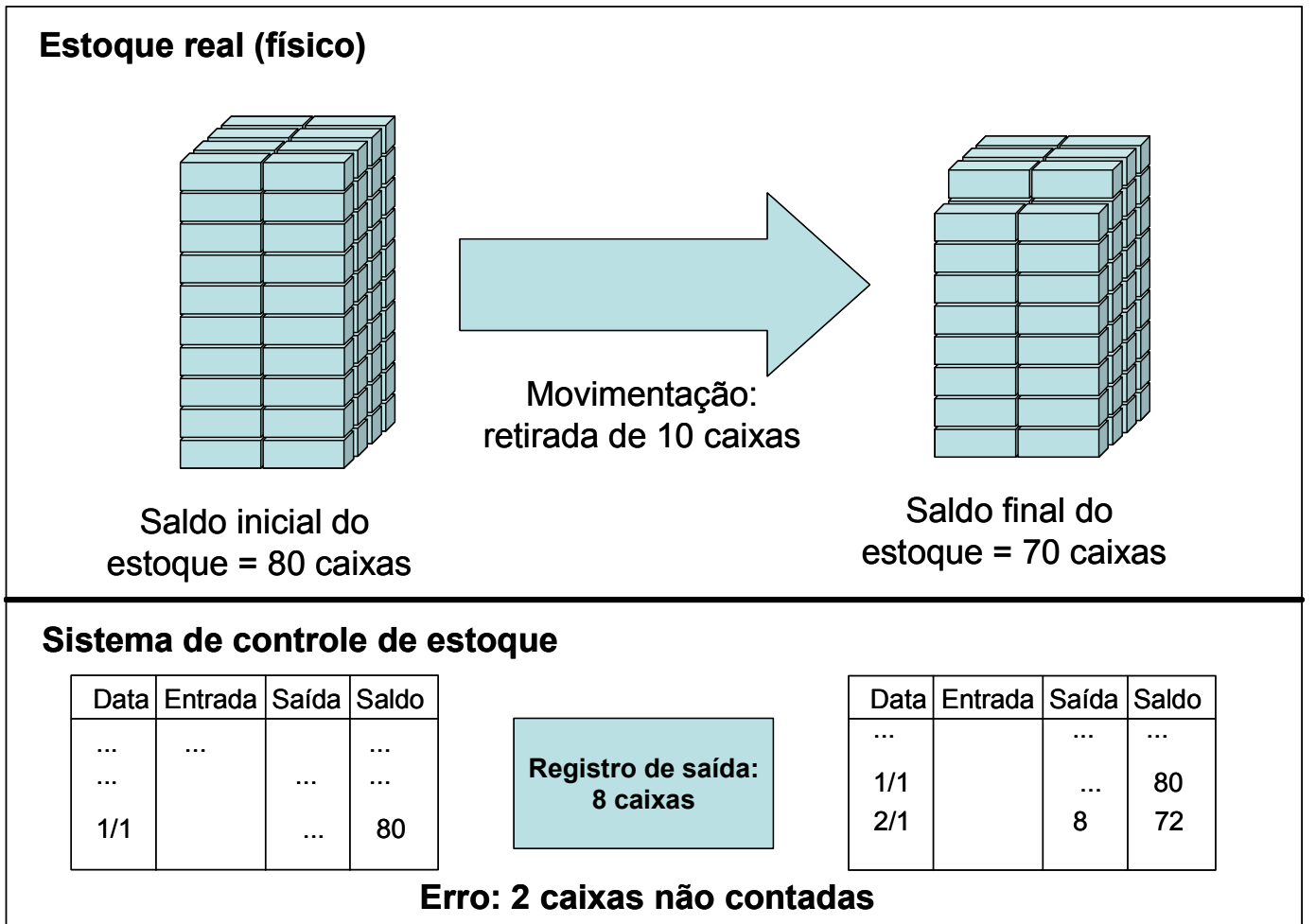

Figura 1 - Controles de estoque real e por um sistema de controle.

As informações (medidas) usadas para representar a acuracidade dos estoques são:

- Inacuracidade (quantidade) de cada produto - Esta medida é o valor absoluto da diferença entre as quantidades apresentadas no estoque real e do sistema ao final do trigésimo dia. Para o cenário é feita uma média dos mil produtos.

- Inacuracidade (proporção) de cada produto - Esta medida divide o valor acima pela quantidade do estoque real, mostrando então a proporção do desvio, em relação ao volume total de todos os produtos. É apresentada a proporção média de inacuracidade e o desvio padrão desta média.

- $\quad$ Saldo diferente - Mede quantos produtos tiveram valores diferentes entre o controle do estoque real e do sistema. 


\subsection{Resultados obtidos}

$\mathrm{Na}$ Tabela 1 são apresentados os resultados obtidos para cada uma das medidas analisadas com as diferentes tolerâncias consideradas, considerando-se inicialmente o cenário com uma probabilidade de ocorrência de erros de $1 \%$.

Tabela 1 - Resultados obtidos com probabilidade de ocorrência de erros de 1\%

\begin{tabular}{|l|l|l|l|}
\hline Medida & Sem tolerância & $\begin{array}{l}\text { Tolerância de } \\
\mathbf{1 \%}\end{array}$ & $\begin{array}{l}\text { Tolerância de } \\
\mathbf{5 \%}\end{array}$ \\
\hline $\begin{array}{l}\text { Inacuracidade } \\
\text { (quantidade) de de } \\
\text { cada produto }\end{array}$ & $\begin{array}{l}\text { Média de 62,8 e } \\
\text { desvio padrão de } \\
305 \text { unidades }\end{array}$ & $\begin{array}{l}\text { Média de 62,4 e } \\
\text { desvio padrão de } \\
\text { 305 unidades }\end{array}$ & $\begin{array}{l}\text { Média de 56,7 e } \\
\text { desvio padrão de } \\
305 \text { unidades }\end{array}$ \\
\hline $\begin{array}{l}\text { Inacuracidade } \\
\text { (proporção) } \\
\text { cada produto de }\end{array}$ & $\begin{array}{l}\text { Média de 5 e e } \\
\text { desvio padrão de } \\
25 \%\end{array}$ & $\begin{array}{l}\text { Média de 5 e } \\
\text { desvio padrão de } \\
25 \%\end{array}$ & $\begin{array}{l}\text { Média de 4 e } \\
\text { desvio padrão de } \\
25 \%\end{array}$ \\
\hline Saldo diferente & $\begin{array}{l}280 \text { produtos com } \\
\text { saldo diferente }\end{array}$ & $\begin{array}{l}230 \text { produtos com } \\
\text { saldo diferente }\end{array}$ & $\begin{array}{l}110 \text { produtos com } \\
\text { saldo diferente }\end{array}$ \\
\hline
\end{tabular}

No caso da medida de inacuracidade por quantidade, os valores obtidos para as situações onde não existe tolerância a erros e onde esta tolerância é de desvios de até 1\% são praticamente iguais: 62,8 e 62,4 unidades, respectivamente. Quando se aumenta a tolerância para erros de até $5 \%$, este valor cai consideravelmente para 56,7 unidades, mostrando uma diferença de 6 unidades. As dispersões destas medidas, representadas pelo desvio padrão, não apresentaram diferenças.

Já a inacuracidade proporcional não apresentou diferenças entre as situações sem tolerância e com tolerância de até 1\%. Esta mesma medida considerada com tolerância de até $5 \%$ apresentou um valor inferior aos demais de $4 \%$ mostrando uma redução de um ponto percentual. Assim como para a medida anterior, não houve variações nas dispersões das medidas. Cabe ressaltar que esta medida mostra a proporção de produtos que apresentam registros sem acuracidade.

A medida que apresentou maior variação foi em relação ao número de produtos com saldos diferentes entre o controle real e do sistema. Na situação sem tolerância a erros, o valor obtido foi de 280 de um total de 1000 produtos (28\%). Nas situações com tolerância, os valores foram de 230 produtos para tolerância de até $1 \%$ e 110 produtos para tolerância até $5 \%$.

$\mathrm{Na}$ Tabela 2 são apresentados os resultados obtidos para cada medida com as diferentes tolerâncias consideradas, considerando-se 0 cenário com uma probabilidade de ocorrência de erros de $10 \%$ em cada registro de movimentação dos estoques.

Tabela 2 - Resultados obtidos com probabilidade de ocorrência de erros de 10\%

\begin{tabular}{|l|l|l|l|l|}
\hline Medida & Sem tolerância & $\begin{array}{l}\text { Tolerância de } \\
\mathbf{1 \%}\end{array}$ & $\begin{array}{l}\text { Tolerância de } \\
\mathbf{5 \%}\end{array}$ \\
\hline $\begin{array}{l}\text { Inacuracidade } \\
\text { (quantidade) de } \\
\text { cada produto }\end{array}$ & $\begin{array}{l}\text { Média de 192,1 e } \\
\text { desvio padrão de } \\
452 \text { unidades }\end{array}$ & $\begin{array}{l}\text { Média de 190,1 e } \\
\text { desvio padrão de } \\
453 \text { unidades }\end{array}$ & $\begin{array}{l}\text { Média de 174,2 e } \\
\text { desvio padrão de } \\
458 \text { unidades }\end{array}$ \\
\hline Inacuracidade & Média de 16 e & Média de 16 e & Média de 15 e \\
\hline
\end{tabular}




\begin{tabular}{|l|l|l|l|}
\hline $\begin{array}{l}\text { (proporção) de } \\
\text { cada produto }\end{array}$ & $\begin{array}{l}\text { desvio padrão de } \\
44 \%\end{array}$ & $\begin{array}{l}\text { desvio padrão de } \\
44 \%\end{array}$ & $\begin{array}{l}\text { desvio padrão de } \\
44 \%\end{array}$ \\
\hline Saldo diferente & $\begin{array}{l}960 \text { produtos com } \\
\text { saldo diferente }\end{array}$ & $\begin{array}{l}850 \text { produtos com } \\
\text { saldo diferente }\end{array}$ & $\begin{array}{l}490 \text { produtos com } \\
\text { saldo diferente }\end{array}$ \\
\hline
\end{tabular}

$\mathrm{Na}$ medida da inacuracidade por quantidades, as variações foram mais acentuadas em comparação ao cenário com menor probabilidade de erros. $\mathrm{Na}$ situação onde não há tolerância a erros, a média foi de 192,1 produtos de diferença entre os controles de estoque real e do sistema. Com uma tolerância de $1 \%$ esta diferença cai para 190,1 unidades, e com uma tolerância de 5\% esta medida assume o valor de 174,2 unidades. As dispersões destas medidas não apresentaram variações significativas.

A medida da inacuracidade proporcional apresentou valores razoavelmente próximos considerando as situações sem tolerância erros e com tolerâncias de 1 e $5 \%$, sendo respectivamente os seguintes valores: 16, 16 e 15\%. As dispersões destas medidas não apresentaram variações.

Para o cenário de $10 \%$ de probabilidade de erros, as medidas da inacuracidade através da diferença de saldos foram bastante acentuadas. $\mathrm{Na}$ situação sem tolerância a erros, foram encontradas diferenças entre o saldo real e do sistema em 960 produtos do total de 1000 produtos. Com uma tolerância de 1\% este valor cai para 850 produtos, e com uma tolerância de $5 \%$ este valor cai ainda mais, para 490 unidades.

\subsection{Análise dos resultados}

$\mathrm{Na}$ seção anterior foram apresentados os resultados das simulações realizadas, e nesta seção os mesmos serão analisados.

Mesmo com uma pequena probabilidade $(1 \%)$ de ocorrência de erros nas movimentações de estoques, a ocorrência de erros nos saldos destes estoques foi significativa. Considerando-se esta probabilidade de ocorrência de erros em 30 movimentações no estoque de cada material analisado, 280 de um total de 1000 produtos apresentaram saldos diferentes entre o controle real e do sistema. Neste cenário de $1 \%$ de probabilidade de ocorrência de erros, a passagem da situação sem tolerância e com tolerância de 1\% não apresentou diferenças significativas, praticamente mantendo os mesmos valores nas medidas de inacuracidade proporcional e pelo número de produtos. A exceção é na medida de quantidade de produtos com saldos diferentes, que cai de 280 para 110 produtos comparando-se as situações sem tolerância e com tolerância de $5 \%$. Isso indica que apenas menos da metade dos produtos com diferença entre os saldos real e do sistema seriam considerados na situação com tolerância, permitindo que o planejamento da produção utilizasse uma informação pouco acurada. A não variação nas dispersões das medidas indica que apesar de ocorrerem pequenas variações na média, os valores unitários não se afastaram dessa média. Em outras palavras, os valores máximos e mínimos observados não se alteraram com a mudança das situações de tolerância aos erros.

A seguir serão feitas análises dos resultados obtidos no cenário de $10 \%$ de probabilidade de ocorrência de erros nos registros da movimentação de estoques. 
Este erro é aparentemente grande, porém não é incompatível com a realidade de muitas empresas (CORREA et alli, 2001). Neste cenário, quase a totalidade dos produtos (960 de 1000) apresentou saldos diferentes entre os controles de estoques real e do sistema. No geral, a mudança das situações de tolerância a erros apresentou variações. A mais significativa destas é na medida da quantidade de produtos com saldos diferentes entre os dois controles. Na situação sem tolerância erros, foram detectados 960 produtos com diferença entre os saldos, e na mudança para as situações de 1 e $5 \%$ de tolerância esta medida cai para 850 e 490 produtos respectivamente. Isso equivale a dizer que mesmo uma pequena tolerância encobre ou oculta muitos problemas. A tolerância de $5 \%$ é aceitável por muitas empresas conforme Corrêa et alli (2001) e na simulação realizada ocultaria praticamente a metade dos problemas de diferença de saldos.

Independentemente do cenário analisado (1 ou $10 \%$ de probabilidade de erros nos registros), a medida da inacuracidade proporcional apresentou variações muito pequenas comparando-se as distintas situações de tolerância aos erros. Já a medida que contabiliza a diferença entre os saldos foi a que apresentou a maior variação.

Cada um dos dois cenários apresentados é uma situação particular do estoque de 1000 produtos. A rigor, as medidas utilizadas deveriam dar visões semelhantes ou pelo menos complementares. Entretanto, pode-se perceber que as informações utilizadas para descrever esta situação são sensivelmente distintas. 0 fato do controle de estoques ter tolerância a erros deveria ser visto como uma situação de exceção onde seria muito complexo ou dispendioso não se considerar nenhuma tolerância. A tolerância oculta distorções que existem, mas não são contidas na informação (as medidas) utilizada. As medidas da inacuracidade pela quantidade mostram a diferença na quantidade de produtos que, na média, existe entre o saldo do controle de estoque real e do sistema. Essa informação fica carente de significado quando isolada da quantidade geral de produtos, e o aumento ou diminuição de uma unidade nesta medida tem que ser interpretada também em comparação à quantidade geral. As medidas proporcionais apresentaram pouca variação mesmo considerando as situações extremas de não haver e de ter a maior tolerância.

\section{CONCLUSÕES}

Este trabalho mostrou que a mesma situação (um controle de estoques) pode ser apresentada de formas diferentes, através do uso de informações distintas. Conforme discutido anteriormente, isso pode trazer incertezas nas decisões tomadas com base nestas informações. Essencialmente foram analisadas três informações sobre a acuracidade de um estoque.

A medida da quantidade média de diferença entre os produtos dá uma visão parcial da realidade, pois precisa ser comparada com o total de produtos e não representa muito bem as variações na tolerância. Por exemplo, uma diferença média de 62,8 unidades tem uma dimensão perante um nível médio de 100 unidades em estoque e outra dimensão completamente diferente perante um nível médio de 10.000 unidades. O mesmo pode ser dito se esta informação é complementada pelo nível total de produtos de todos os estoques. Assim, esta informação não deve ser 
usada de forma isolada e serve apenas de referência, não sendo indicada para se ter uma visão da acuracidade (ou inacuracidade) de um estoque.

A informação da medida proporcional das diferenças entre os controles de estoque real e do sistema incorpora a quantidade total para se calcular a proporção. Assim, esta informação é mais sensível à quantidade de cada produto. No caso de muitos produtos com quantidades muito variadas esta medida ainda pode não dar uma boa visão da acuracidade geral.

A última informação utilizada - a quantidade de produtos com diferença entre os saldos - dá uma visão muito clara da existência da falta de acuracidade, principalmente se acompanhada do número total de produtos. Por outro lado, esta informação não mostra a dimensão da falta de acuracidade em cada controle de estoques.

Pode-se concluir que o gestor de um estoque que necessite conhecer a falta de acuracidade do mesmo deve utilizar mais de uma informação para esta finalidade. No caso das medidas analisadas neste trabalho sugere-se a utilização conjunta das medidas de diferença proporcional e quantidade de produtos com diferença, complementadas com a informação do volume total de produtos e da média dos volumes dos estoques de todos os produtos. Como pesquisas futuras serão analisadas ainda outras medidas com esta finalidade, principalmente aquelas que representem de alguma forma as perdas financeiras com a falta de acuracidade nos estoques.

\section{AGRADECIMENTOS}

O autor agradece os auxílios recebidos do Conselho Nacional de Desenvolvimento Científico e Tecnológico - CNPq.

\section{REFERÊNCIAS}

BALLARD, R. L. Methods of inventory monitoring measurement. Logistics Information Management, v. 9, n. 3, 1996.

BASINGER, K. L. Impact of Inaccurate Data on Supply Chain Inventory Performance. Tese (doutorado), The Ohio State University, 2006.

BAZERMAN, M. H. Processo decisório. Editora Campus, Rio de Janeiro, 2004.

BROWN, K. L.; INMAN, R. A.; CALLOWAY, J. A. Measuring the effects of inventory inaccuracy in MRP inventory and delivery performance. Production Planning \& Control, v. 12, n. 1, 2001.

BUXEY, G. Reconstructing inventory management theory. International Journal of Operations \& production Management, v. 26, n. 9, 2006. 
CHENGALUR-SMITH, I. N.; BALLOU, D. P.; PAZER, H. L. The impact of data quality information on decision making: an exploratory analysis. IEEE Transactions on Knowledge and Data Engineering, v. 11, n. 6, 1999.

CORREAA, H.; GIANESI, I.; CAON, M. Planejamento, programação e controle da produção. Editora Atlas, 2001.

DEHORATIUS, N.; RAMAN, A. Inventory record inaccuracy: An Emprical Analysis. Management Science, v. 54, n. 4, p. 627-641, 2008.

DROHOMERETSKI, E. Um estudo do impacto das formas de controle de inventário na acuracidade de estoque. Dissertação de mestrado. Pontifícia Universidade Católica do Paraná, Curitiba, 2009.

FAVARETTO, F. Qualidade da informação no planejamento detalhado da produção. Anais do VI Congresso Nacional de Excelência em Gestão - CNEG, Niterói-RJ, 2010.

FELDMANN, M.; MÜLLER, S. An incentive scheme for true information providing in supply chains. OMEGA - The International Journal of Management Science, v. 31, 2003.

FLEISCH, E.; TELLKAMP, C. The impact of inventory inaccuracy on retail supply chain performance: a simulation study. International Journal of Production Economics, v. 95, n. 3, 2003.

GUIMARÃES, E. M. P.; ÉVORA, Y. D. M. Sistema de informação: instrumento para a tomada de decisão no exercício da gerência. Ciência da Informação, v. 33, n. 1, 2004.

HUSCHKA, K. Using Statistical Process Control to Monitor Inventory Accuracy. Dissertação (mestrado). Kansas State University, 2009.

KLEIN, B. D. Detecting errors in data: clarification of the impact of base rate expectations and incentives. Omega, v. 29, 2001.

REID, A.; CATTERALL, M. Invisible data quality issues in a CRM implementation. Database Marketing \& Customer Strategy Management, v. 12, n. 4, 2005.

UCKUN, C; KARAESNEN, F.; SAVAS, S. Investment in improved inventory accuracy in a decentralized supply chain. International Journal of Production Economics, n._113, p. 546-566, Junho, 2008.

WALLER, M. A. ; NACHTMANN, H.; HUNTER, J. Measuring the impact of inaccurate inventory information on a retail outlet. The International Journal of Logistics Management, v. 17 n. 3, p. 355-376, 2006.

ZHANG, C.; TAN, G.; ROBB, D. J.; ZHENG, X. Sharing shipment quantity information in the supply chain. OMEGA - The International Journal of Management Science, Vol. 34, 2006. 